\title{
An Improved Method for Measuring Quantitative Resistance to the Wheat Pathogen Zymoseptoria tritici Using High-Throughput Automated Image Analysis
}

\author{
Ethan L. Stewart, Christina H. Hagerty, Alexey Mikaberidze, Christopher C. Mundt, \\ Ziming Zhong, and Bruce A. McDonald
}

First, fifth, and sixth authors: Plant Pathology, Institute of Integrative Biology, ETH Zürich, Zürich; second and fourth authors: Department of Botany and Plant Pathology, Oregon State University, Corvallis; and third author: Theoretical Biology, Institute of Integrative Biology, ETH Zürich.

Accepted for publication 19 March 2016.

\begin{abstract}
Stewart, E. L., Hagerty, C. H., Mikaberidze, A., Mundt, C. C., Zhong, Z., and McDonald, B. A. 2016. An improved method for measuring quantitative resistance to the wheat pathogen Zymoseptoria tritici using high-throughput automated image analysis. Phytopathology 106:782-788.

Zymoseptoria tritici causes Septoria tritici blotch (STB) on wheat. An improved method of quantifying STB symptoms was developed based on automated analysis of diseased leaf images made using a flatbed scanner. Naturally infected leaves $(n=949)$ sampled from fungicide-treated field plots comprising 39 wheat cultivars grown in Switzerland and 9 recombinant inbred lines (RIL) grown in Oregon were included in these analyses. Measures of quantitative resistance were percent leaf area covered by lesions, pycnidia

size and gray value, and pycnidia density per leaf and lesion. These measures were obtained automatically with a batch-processing macro utilizing the image-processing software ImageJ. All phenotypes in both locations showed a continuous distribution, as expected for a quantitative trait. The trait distributions at both sites were largely overlapping even though the field and host environments were quite different. Cultivars and RILs could be assigned to two or more statistically different groups for each measured phenotype. Traditional visual assessments of field resistance were highly correlated with quantitative resistance measures based on image analysis for the Oregon RILs. These results show that automated image analysis provides a promising tool for assessing quantitative resistance to $Z$. tritici under field conditions.
\end{abstract}

Wheat is the most important food crop in Europe and second most important worldwide after rice, with an average annual global production of $>600$ million tons (http://faostat3.fao.org/browse/Q/ QC/E). Zymoseptoria tritici (formerly Mycosphaerella graminicola) is the most damaging wheat pathogen in Europe (Jørgensen et al. 2014; O'Driscoll et al. 2014) and is considered an important fungal pathogen worldwide (Dean et al. 2012). Symptoms typically include leaf chlorosis 10 to 14 days after infection that develops into necrotic lesions. Small, dark, asexual fruiting structures called pycnidia begin to develop soon after the onset of necrosis. Pycnidiospores are exuded from the pycnidia during periods of high humidity and are dispersed throughout the crop canopy by rain splash. The asexual cycle is the primary source of plant damage and may occur multiple times during a growing season. Currently, there is no completely effective control strategy for Z. tritici. The main control methods are fungicide treatments and planting resistant varieties. Even with the combined use of resistant cultivars and regular fungicide treatments, yield losses of 5 to $10 \%$ can be expected (Fones and Gurr 2015). In Europe, approximately 1 billion euros is spent on fungicides annually to control Z. tritici (Torriani et al. 2015). With resistance to fungicides becoming an increasing problem, greater focus must be placed on exploiting genetic resistance as a control method (Brown et al. 2015; Orton et al. 2011). Given that major gene resistance is prone to failure (Mundt

Corresponding author: E. Stewart; E-mail address ethan.stewart@usys.ethz.ch

*The $\boldsymbol{e}$-Xtra logo stands for "electronic extra" and indicates that nine supplementary figures and three supplementary tables are published online.

http://dx.doi.org/10.1094/PHYTO-01-16-0018-R

(C) 2016 The American Phytopathological Society
2014) and most virulence is quantitative (Stewart and McDonald 2014; Zhan et al. 2007), successful breeding strategies are likely to rely on quantitative resistance. Twenty-one resistance genes have been identified in wheat that confer mainly qualitative resistance to $Z$. tritici and show strong host-isolate specificity. In addition, 89 genomic regions have been identified that confer low levels of quantitative (partial) resistance to a wide range of isolates (Brown et al. 2015).

Accurate phenotypic data are needed to more effectively develop and deploy disease-resistant varieties. Researchers need accurate measurements of disease to evaluate the effects of different types of resistance on the pathogen under controlled environmental conditions. Breeders would benefit from more accurate measurements to choose among resistant lines in breeding nurseries and field trials. National agricultural research agencies need accurate measurements of resistance under field conditions to generate lists of recommended cultivars for farmers. In addition, accurate and precise measurements of disease development are useful for measuring and validating the effectiveness of fungicides and biological control agents.

Accurately scoring subtle differences in quantitative resistance showing a continuous distribution is more difficult than scoring major gene resistance that shows a largely binomial distribution. High-throughput systems are needed to screen multiple plant lines, possibly over multiple time points. Digital image analysis provides an accurate way to measure levels of plant disease as well as other traits of interest. Image-based phenotyping is increasingly used in experimental systems but has not been widely adopted in breeding programs. We previously described a method for quantifying disease in the $Z$. tritici-wheat pathosystem based on high-throughput image analysis using a batch-processing macro in the open source image processing software ImageJ (Rasband 1997-2015; Stewart and McDonald 2014). This method was shown to be more accurate and reproducible than visual estimates in a controlled 
greenhouse seedling assay. Two shortcomings of this method were difficulties in implementation for users not familiar with ImageJ macros and variations in camera and light set-ups, making comparisons between experiments and groups difficult. Conventional flatbed scanners are inexpensive and offer consistent settings and light levels. Scanners have previously been used to phenotype rice root architecture (Kato et al. 2010), microbial growth in microtiter plates (Gabrielson et al. 2002), Arabidopsis leaf morphology (Maloof et al. 2013), and Cladosporium fulvum symptoms in tomato (Abd-El-Haliem 2012).

We adapted our previously described camera-based method to utilize flatbed scanners and improve the ease of acquisition of digital images of symptoms caused by Z . tritici. We then used this method to measure quantitative resistance to $Z$. tritici under field conditions among 39 naturally infected wheat cultivars in Switzerland and 9 naturally infected recombinant inbred lines (RIL) in Oregon. Our findings indicate that automated image analysis can provide a powerful tool to differentiate among different degrees and forms of quantitative resistance under field conditions, suggesting that this method may be useful for breeding quantitative resistance to $Z$. tritici.

\section{MATERIALS AND METHODS}

Several changes were made to the method reported previously (Stewart and McDonald 2014). To address the issue of variation among labs due to differences in lighting and camera setups, the method was adapted to use a flatbed scanner instead of a camera. This allows the settings to be more consistent between experiments and laboratories. In addition, the ImageJ macro was modified to make it more user friendly and easier to customize (Supplementary Fig. S1). A Linux shell script (Supplementary Fig. S2) reads a text file containing a list of sample names as input and creates a .pdf file with the open source typesetting software LaTeX (LaTeX3 Project Team 2015) containing pages on which to mount infected leaves. Each printed page contains fixed reference points used to set the image scale and boxes within which to mount the leaves. Each box contains one of the sample names provided in the text file in humanreadable text as well as encoded as a $\mathrm{QR}$ code. After mounting the leaves, the pages are scanned using a conventional flatbed scanner at a resolution of 1,200 dpi and images are saved in .tiff or .jpg format. After scanning, the images are processed using ImageJ via a batch-processing macro, described below.

Several plugins were used or modified to achieve greater usability of the macro (Supplementary Figs. S3 to S7). The macro automatically checks whether these plugins are present and compiles them if necessary. Before running the image analysis, the new macro produces several pop-up boxes asking the user for various inputs. Here, the user specifies various parameters such as color threshold values for lesion detection and size and shape descriptors for pycnidia detection. These inputs are stored as variables and used throughout the macro, eliminating the need to manually customize the macro code. Macro settings are saved and can be used in subsequent runs of the macro without having to reenter the parameter values. Each leaf is analyzed individually, with the sample name for each leaf read from the QR code. A customized results table was also added that outputs summary data on total leaf area, lesion area, percent leaf area covered by lesions (PLACL), pycnidia count, pycnidia per square centimeter of leaf area, pycnidia per square centimeter of lesion leaf area, mean pycnidia size, and mean pycnidia gray value, as well as sample name for each leaf. By choosing different color threshold settings, the macro will either measure leaf necrosis (brown senesced tissue) or leaf lesions (brown senesced tissue, chlorotic tissue, and any other nongreen leaf tissue). Leaf lesions were used in this study. Pycnidia gray values are obtained by converting the RGB image into an 8-bit grayscale image and calculating the mean gray value of the pixels making up each pycnidium. The grayscale ranges from 0 (black) to 255 (white) and can be used as a proxy for degree of melanization in Z. tritici (Lendenmann et al. 2014). An overlay image for each leaf is produced with the total leaf area, lesion area, and each pycnidium visibly outlined. Coherent step-by-step instructions to implement the analysis were also written (Supplementary Fig. S8).

Validation using naturally infected leaf samples from field experiments. Leaves were collected from field plots naturally infected with $Z$. tritici at the Field Phenotyping Platform (http://www.kp.ethz.ch/infrastructure/FIP.html) at the Eschikon Field Station of ETH Zürich, Switzerland (coordinates 47.449683, 8.682461) and at the Oregon State University Hyslop Farm Field Laboratory north of Corvallis, OR (coordinates 44.633400, -123.193957 ). Swiss plots measuring $0.75 \mathrm{~m}$ wide by $1.5 \mathrm{~m}$ long were planted to winter wheat cultivars varying in resistance to Z. tritici in a randomized block design with two blocks, with each cultivar represented in both blocks. Plots received four fungicide treatments throughout the growing season, one in April (Input; Bayer), two in May, (Gladio; Syngenta and Aviator Xpro; Bayer), and a fourth in June (Proline; Bayer). In all, 30 Swiss cultivars were sampled along with 9 cultivars chosen because of differences in their stomatal density. Existing disease ratings for 21 of the Swiss cultivars were taken from the Swiss Granum recommended varieties lists (Courvoisier et al. 2015) published between 2008 and 2015. On 30 June 2015, 20 leaves were collected from each cultivar, with 10 leaves sampled from each block. Nonsenescent leaves exhibiting obvious Septoria tritici blotch (STB) lesions were collected at random from the topmost infected leaf layer throughout each plot, typically originating from one or two leaves below the flag leaf on a sampled plant. Infected leaves were placed in paper envelopes, kept on ice in the field, and stored at $4^{\circ} \mathrm{C}$ overnight before mounting on plain paper containing reference marks and sample names, as described above. Absorbent paper was placed between each sheet of mounted leaves and sheets were pressed with approximately $5 \mathrm{~kg}$ at $4{ }^{\circ} \mathrm{C}$ overnight prior to scanning. Pages were scanned at a resolution of 1,200 dpi using a flatbed scanner (EPSON XP-810) and the resulting images were saved in tif format.

Oregon leaves were sampled from the 'Einstein' $\times$ 'Tubbs' RIL population (Vazquez et al. 2015), which consists of 259 RILs varying in resistance to $Z$. tritici and other pathogens. The population was planted in early October 2014. Similarly to the Swiss plots, the Oregon plots were planted in a randomized block design with two blocks. Each plot consisted of three rows that were $3.0 \mathrm{~m}$ long, with $20.3 \mathrm{~cm}$ between rows. All plots received four applications of azoxystrobin fungicide (Quadris; Syngenta) over the course of the season to prevent establishment of stripe rust (Puccinia striiformis) and to suppress development of other fungal diseases. The Oregon $Z$. tritici population is predominately resistant to azoxystrobin at this point in time (Hagerty and Mundt 2015) and the STB epidemic progressed normally despite the fungicide applications. Of the 259 RIL lines, 9 were chosen for analysis based on visible differences in resistance to $Z$. tritici coupled with late maturity. Field disease ratings for the Oregon lines were taken on 5 June 2015. Each plot was assigned a disease severity rating between 0 and $100 \%$. On 10 June 2015, 24 leaves were collected from each of the nine selected lines, representing four flag leaves evenly spaced from each row of each plot. Flag leaves exhibiting $Z$. tritici symptoms that were not yet senesced were collected without regard to the level of disease. Infected leaves were placed between pages of a heavy textbook in the field, and stored on a lab bench overnight before mounting on plain paper the following day. Mounted leaves were kept flat, cool, and dry until October 2015, when leaves were remounted on paper containing reference marks and sample names, as described above. Oregon leaves were scanned immediately following the remounting using a flatbed scanner (Canon CanoScan LiDE 700F) and the images were saved in .jpg format.

Image analysis. Images were analyzed using the ImageJ macro described above (macro settings given in Supplementary Fig. S9). For each leaf, the macro measured total leaf area, total lesion area, number 
of pycnidia, mean size of pycnidia, and pycnidia gray value. PLACL was calculated by dividing the lesion area by the total leaf area. Pycnidia density per square centimeter of leaf area and per square centimeter of lesion area was calculated by dividing the number of pycnidia by the total leaf area and total lesion area, respectively.

Based on the image analysis results, 18 representative leaves were selected across the range of pycnidia counted, ranging from 0 to 1,000 per leaf in increments of approximately 50 pycnidia. First, the data were ordered according to the total number of pycnidia counted per leaf. Then, the leaves containing the number of pycnidia closest to a multiple of 50 were selected for further analysis. The pycnidia from these leaves were counted manually using the multipoint selection tool in ImageJ.

The same leaves that were used for the comparison between scanner and manual counts were rescanned with two different scanners (EPSON XP-810 and Canon CanoScan LiDE 220) using the above settings to test for variation between scanners. Gray values of the black reference points printed on each page were measured using the measure command in ImageJ. Similarly, three areas on each page containing neither printed matter nor leaves were measured per image to gain a measure of the gray value from blank areas.

Data analyses. All analyses were carried out in base R (R Core Team 2012) unless specified differently. Mean values plus standard deviations were calculated for each phenotype on each host. The effect of cultivar on phenotype and comparisons of treatments were performed using the Kruskal-Wallis test and multiple comparisons of treatments from the Agricolae package (de Mendiburu 2014) using a Bonferroni correction for multiple comparisons. Differences between blocks were calculated with a Wilcoxon signed rank test. Correlations were performed with Pearson's product moment correlation coefficient. Correlations between phenotypes were made between values for each leaf. Correlations between field disease ratings and image analysis in the Oregon data set were made between visual scores for each plot and the mean value per plot from the image analysis. In the case of multiple correlations, $P$ values were corrected using Holm's correction. Differences in variance between phenotypes from the two datasets were calculated with a Fligner-Killeen test. Lin's concordance correlation coefficient (Lin 1989) between actual values and values from image analysis was calculated as previously described (Stewart and McDonald 2014) using the epi.ccc function in the epiR package (Stevenson et al. 2013). Differences between scanners were calculated using a Mann-Whitney test.

\section{RESULTS}

From the image analysis output, the phenotypes PLACL, number of pycnidia per leaf, pycnidia per square centimeter of leaf, pycnidia per square centimeter of lesion, mean pycnidia size, and mean pycnidia gray value were considered the traits most relevant to assessing quantitative resistance and were used for further analysis.

All phenotypes showed a continuous distribution in both field sites (Fig. 1). All phenotypes showed a similar distribution in the two datasets, except PLACL, which showed a U-shaped distribution in the Oregon lines. The mean values of PLACL for each cultivar in
PLACL

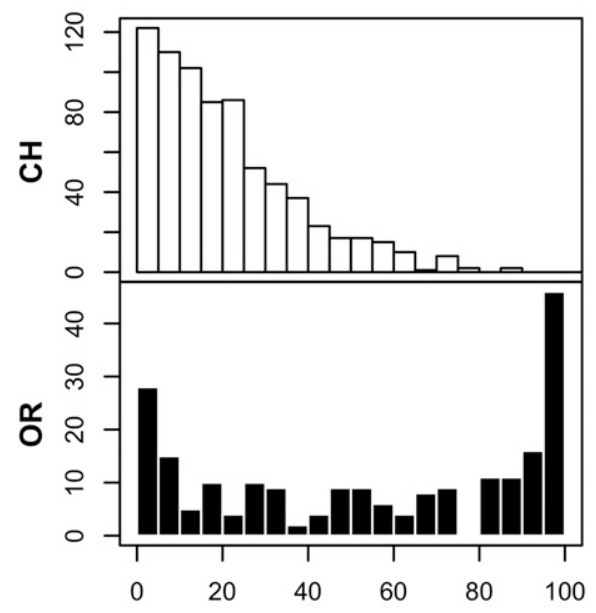

Pycnidia per $\mathrm{cm}^{2}$ Leaf

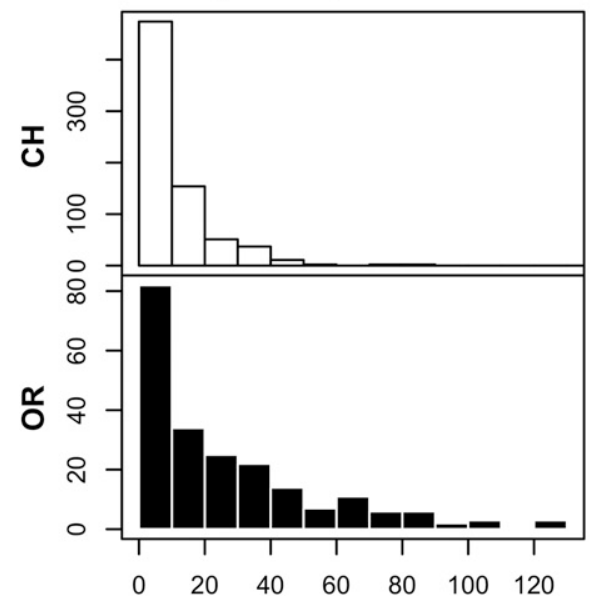

Pycnidia Count

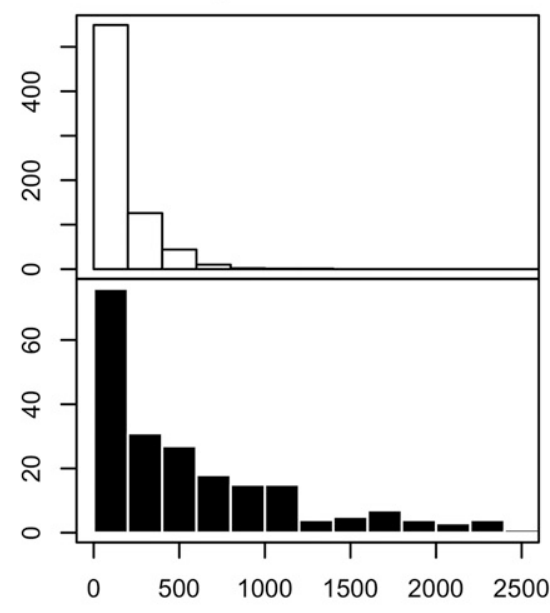

Pycnidia per $\mathrm{cm}^{2}$ Lesion

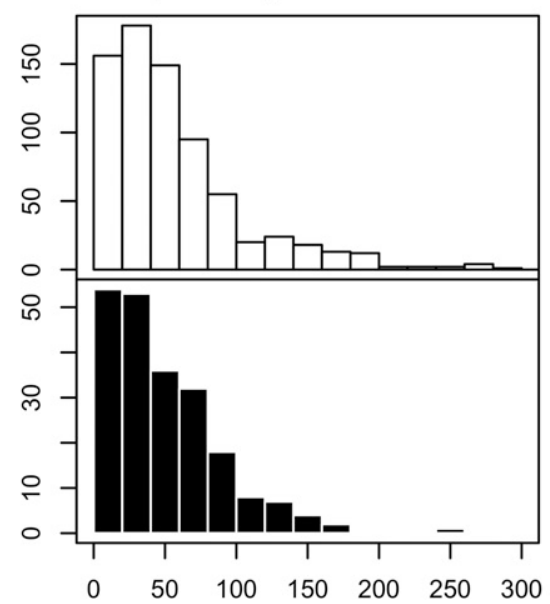

Pycnidia Area

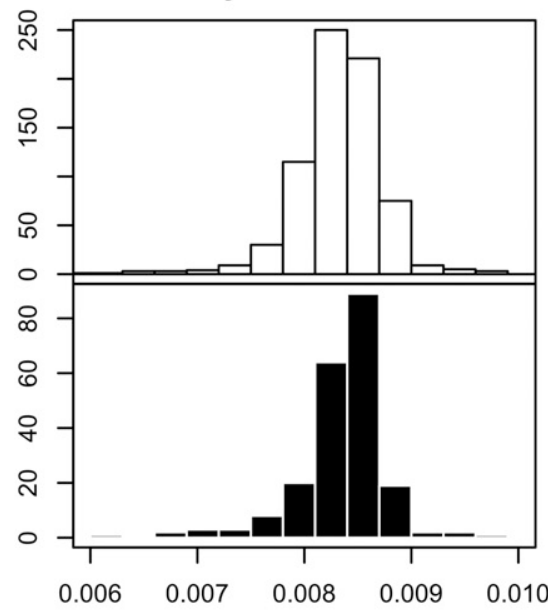

Pycnidia Grey Value

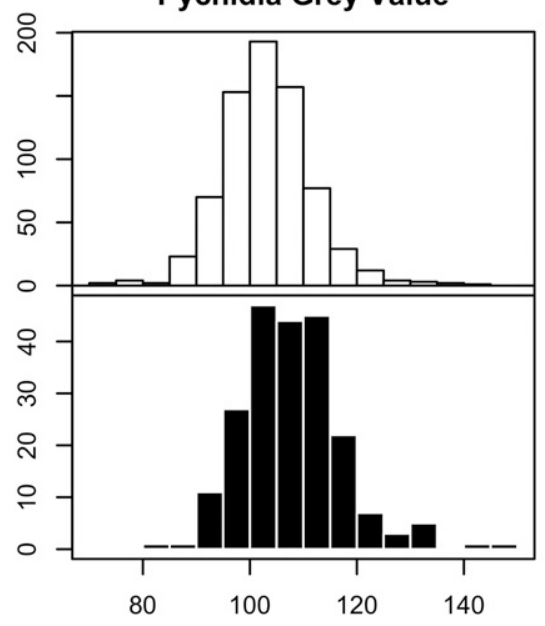

Fig. 1. Frequency histograms of Zymoseptoria tritici virulence phenotypes in naturally infected field plots measured using automated image analysis from Switzerland (CH, white bars) and Oregon (OR, black bars). 
Switzerland ranged from 11.3 to $34.6 \%$. Total number of pycnidia per leaf ranged from 40 to 362 , with pycnidia density within lesions ranging from 14.5 to 128 pycnidia/ $\mathrm{cm}^{2}$ (Supplementary Table S1). The mean number of pycnidia per leaf was 149 (Table 1). Block had a significant effect on PLACL $(\mathrm{W}=77097, P=0.005)$ and pycnidia per square centimeter of leaf $(\mathrm{W}=74022, P=0.017)$ but not on the other phenotypes.

In the Oregon dataset, overall levels of disease were higher, with PLACL ranging from 14.5 to $93.5 \%$. Total number of pycnidia per leaf ranged from 52 to 1471 , with pycnidia density within lesions ranging from 28.3 to $65.5 \mathrm{pycnidia} / \mathrm{cm}^{2}$. The mean number of pycnidia per leaf was 640 (Table 1). No differences between blocks were observed in the Oregon dataset.

Plant genotype had a significant effect on all phenotypes in both locations (Table 2). There was a significant difference between the genotypes showing the highest and lowest values for all phenotypes. Lines and cultivars could be grouped into two or more significantly different groups for all phenotypes in both locations (Supplementary Table S2).

There was a significant, positive correlation between PLACL and pycnidia count and between PLACL and pycnidia per square centimeter of leaf in both the Oregon $\left(r^{2}=0.45, P<0.001\right.$ and $r^{2}=$ $0.46, P<0.001$, respectively) and Swiss $\left(r^{2}=0.31, P<0.001\right.$ and $r^{2}=0.36, P<0.001$, respectively) populations.

Variance was significantly higher in the Oregon dataset for PLACL $\left(\chi^{2}=246.55, P<0.001\right)$, pycnidia count $\left(\chi^{2}=324.19, P<\right.$ $0.001)$, and pycnidia per square centimeter of leaf $\left(\chi^{2}=183.75, P<\right.$ $0.001)$. There was no difference in variance for mean pycnidia size, pycnidia per square centimeter of lesion, or pycnidia gray value between the two datasets.

Visual ratings made in the field in Oregon were significantly correlated with PLACL $\left(r^{2}=0.67, P<0.001\right)$, pycnidia count $\left(r^{2}=\right.$ $0.63, P<0.001)$, and pycnidia per square centimeter of leaf $\left(r^{2}=0.58\right.$, $P<0.001)$ from the image analysis. In the Swiss population, there was no visible association between published cultivar disease ratings and the phenotypes observed from image analysis. Due to the unbalanced numbers of cultivars found within each disease rating (Supplementary Table S3), this association could not be tested statistically.

TABLE 1. Summary of Septoria tritici blotch resistance phenotypes measured by image analysis ${ }^{\mathrm{a}}$

\begin{tabular}{lrrrrr}
\hline & \multicolumn{2}{c}{ Switzerland } & & \multicolumn{2}{c}{ Oregon } \\
\cline { 2 - 3 } \cline { 5 - 6 } Phenotype & Mean & SD & & Mean & \multicolumn{1}{c}{ SD } \\
\hline Leaf area covered by lesions $(\%)$ & 20.7 & 16.6 & & 54.7 & 36.1 \\
Pycnidia count & 149.3 & 158.2 & & 640.6 & 725.0 \\
Mean pycnidia size $\left(\mathrm{mm}^{2} \times 10^{-3}\right)$ & 8.3 & 0.6 & 8.3 & 0.4 \\
Pycnidia per square centimeter of leaf & 10.3 & 11.2 & & 27.3 & 28.9 \\
Pycnidia per square centimeter of lesion & 57.2 & 50.9 & & 51.1 & 44.2 \\
Pycnidia gray value & 103.1 & 9.2 & & 107.9 & 9.2 \\
\hline
\end{tabular}

a Values are mean and standard deviation (SD) for leaves sampled from 39 wheat cultivars from Switzerland $(n=733$ leaves) and 9 wheat recombinant inbred lines from Oregon ( $n=216$ leaves).

${ }^{\mathrm{b}}$ Grayscale ranges from 0 (black) to 255 (white).

TABLE 2. Kruskal-Wallis test summary of wheat cultivar/genotype effect on Septoria tritici blotch resistance phenotypes measured on 39 wheat cultivars from Switzerland and 9 wheat recombinant inbred lines from Oregon

\begin{tabular}{lrrrrrr}
\hline & \multicolumn{2}{c}{ Switzerland } & & \multicolumn{2}{c}{ Oregon } \\
\cline { 2 - 3 } \cline { 6 - 7 } Phenotype & \multicolumn{1}{c}{$\mathrm{W}$} & \multicolumn{1}{c}{$P$ value } & & $\mathrm{W}$ & & $P$ value \\
\hline Leaf area covered by lesions $(\%)$ & 72.8 & 0.001 & & 126.5 & $<0.001$ \\
Pycnidia Count & 188.5 & $<0.001$ & & 130.1 & $<0.001$ \\
Mean pycnidia size & 104.6 & $<0.001$ & & 22.0 & 0.005 \\
Pycnidia per square centimeter of leaf & 177.4 & $<0.001$ & & 124.1 & $<0.001$ \\
Pycnidia per square centimeter of lesion $^{171.6}$ & $<0.001$ & & 44.7 & $<0.001$ \\
Pycnidia gray value $^{\mathrm{a}}$ & 183.9 & $<0.001$ & & 38.5 & $<0.001$ \\
\hline
\end{tabular}

${ }^{a}$ Grayscale ranges from 0 (black) to 255 (white).
Pycnidia counts obtained with the ImageJ analysis were compared with absolute values obtained using manual counts. There was a strong agreement (Lins concordance coefficient $\left[\rho_{c}\right]=0.94$ ) between the automated image analyses and the human-curated counts (Fig. 2). Image analysis was both accurate $\left(C_{b}=0.99\right)$ and precise $(r=0.95)$ when compared with absolute values. For total leaf area and lesion area, overlay images generated by the image analysis were checked manually for accuracy and were deemed to be accurate.

There was no difference between scanners for any of the phenotypes except pycnidia gray values, which were significantly darker $(\mathrm{W}=44.5, P=0.0002)$ from the Epson scanner, with a mean gray value of 98.5 over all pycnidia measured compared with a mean value of 110.9 for the Canon scanner. Black areas from the Epson scanner were significantly darker $(\mathrm{W}=1, P=0.004)$ compared with the Canon scanner, with a mean gray value of 56.1 versus 62.2 for the Canon. However, white areas from the Epson scanner were significantly lighter $(\mathrm{W}=81, P<0.001)$, with a mean gray value of 238.3 compared with 234.8 from the Canon scanner.

The time needed to scan each page, representing eight leaves, was approximately $2 \mathrm{~min}$. The time needed to analyze the 140 pages of leaves from the Swiss dataset was 6 h 15 min of computational time on a standard desktop computer.

\section{DISCUSSION}

An automated image analysis method developed to measure virulence-associated traits in $Z$. tritici was modified to assess quantitative resistance to STB in naturally infected, genetically diverse populations of wheat grown on two continents. The Swiss population was composed of a highly diverse set of popular wheat cultivars grown commercially in Switzerland. The Oregon population was composed of a series of experimental RILs based on a cross between two wheat cultivars known to differ for resistance to $Z$. tritici and chosen to represent different degrees of field resistance based on standardized disease rating scores. Automated image analysis was previously used to measure quantitative virulence traits among $Z$. tritici isolates under greenhouse conditions (Stewart and McDonald 2014) but this is the first application of this method to

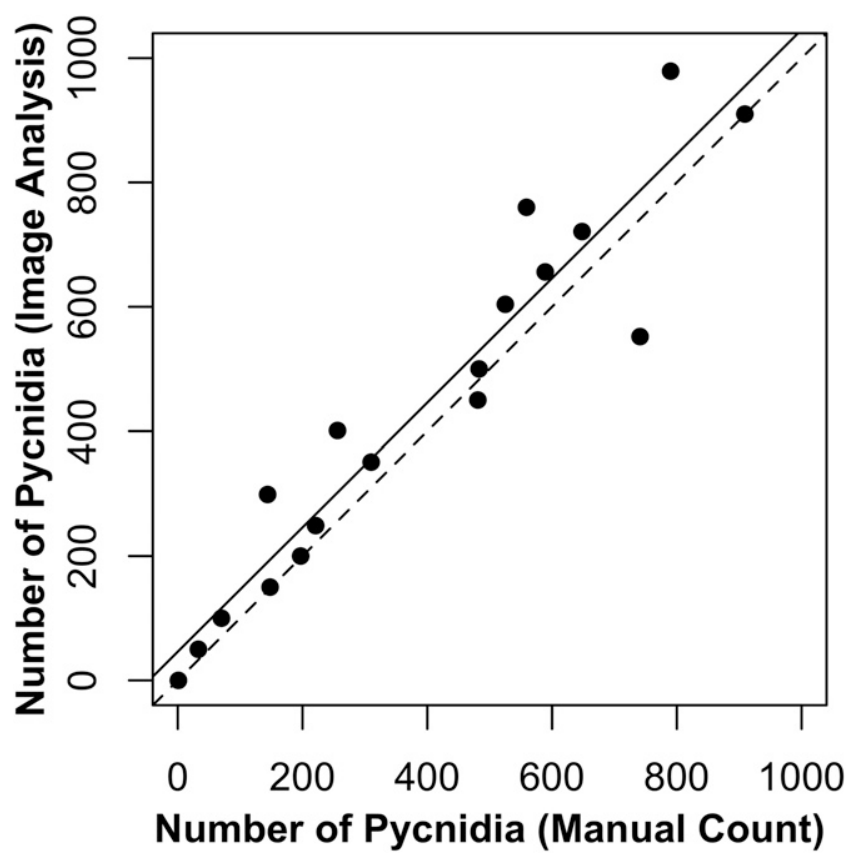

Fig. 2. Agreement between Zymoseptoria tritici pycnidia counts made by image analysis and true values based on manual counts. Dashed line denotes line of perfect concordance and solid line is line of best fit. Lins concordance coefficient $\left(\rho_{c}\right)=0.94$, accuracy $\left(C_{b}\right)=0.99$, and precision $(r)=0.95$. 
wheat plants naturally infected by $Z$. tritici in the field. The method allows several different traits associated with quantitative resistance to be measured independently. Here, we were able to measure PLACL, pycnidia size, pycnidia density over the entire leaf and within lesions, number of pycnidia per leaf, and pycnidia gray values.

The advent of low-cost digital cameras and other sensor technologies (Mahlein 2016) allowed image analysis to become more widely used in plant pathology (Bock et al. 2010; Mutka and Bart 2014). Several software programs designed specifically to use digital images to measure plant disease symptoms on desktop computers (e.g., ASSESS) (Lamari 2002) and, more recently, on mobile devices (e.g., Leaf Doctor) (Pethybridge and Nelson 2015) or using web-based tools (Green et al. 2012) are already available. General image analysis software can also be used to measure plant disease (Abd-El-Haliem 2012; Kwack et al. 2005). Dedicated disease-measuring software has the advantage of being easy to use and requires little or no knowledge of image analysis techniques but often lacks the flexibility needed to measure symptoms other than leaf lesions and, typically, lacks the ability to automatically analyze many images in a high-throughput manner. In contrast, generalpurpose image analysis software has the capacity to detect more complex symptoms and to program high-throughput work flows but also requires some knowledge of image analysis techniques and programming or scripting languages. Scripts written for ImageJ provide the capacity to measure complex disease symptoms in an automated, high-throughput manner that can be implemented by a user with little or no prior knowledge of the underlying image analysis techniques.

There was no evidence for a binary resistant-susceptible phenotype in either of the datasets. All phenotypes showed a continuous distribution, providing further evidence that pathogen virulence and host resistance are usually quantitative traits in natural field infections of $Z$. tritici. PLACL showed a skew to the extremes of the distribution in the Oregon lines. In this case, a subset of nine RIL lines was chosen to represent a broad range of disease levels but it appears that more plants showed symptoms at the extremes of the disease spectrum than in the middle. In the Swiss cultivars, a more continuous distribution in quantitative resistance was observed.

An overall correlation was found between PLACL and total number of pycnidia per leaf and pycnidia per square centimeter of leaf in both datasets. However, there were some instances where lines and cultivars showed relatively high levels of PLACL coupled with low numbers of pycnidia and vice versa. In several cases, wheat cultivars (Switzerland) and experimental lines (Oregon) showed different rankings for the different phenotypes. For example, in Switzerland, PLACL was significantly higher in 'Caphorn' than 'Magno' but there was no significant difference between pycnidia density within lesions for the same cultivars. Conversely, 'Cambrena' had significantly higher pycnidia density within lesions than 'Nirvana' but PLACL was not significantly different. Similar patterns were observed in Oregon lines. This indicates that cultivars may interact differently with the local pathogen population to produce different symptoms. Host specificity is well documented in Z. tritici (Cowger and Mundt 2002; Zhan et al. 2002). From these findings, it appears that the same pathogen population can produce different symptoms on different hosts under field conditions, suggesting that different symptoms may reflect different mechanisms of resistance operating in the field. Numerous resistance genes have been identified in wheat (Brown et al. 2015). Under the gene-for-gene paradigm, each resistance gene would interact with a counterpart in the pathogen. We speculate that different combinations of quantitative resistance genes in the host and quantitative virulence genes in the pathogen combine to produce the observed quantitative resistance phenotypes.

There are numerous ways to measure resistance to $Z$. tritici, as described here and elsewhere (Suffert et al. 2013), though resistance is most commonly measured based on visible leaf damage (i.e., based on percentage of leaf area covered by lesions or necrosis). It is possible that using the additional measures of virulence presented here could identify new resistance genes that affect different pathogen traits, such as pycnidial formation or pycnidial size, but this will require further investigation. We postulate that larger pycnidia contain more or larger pycnidiospores, thus giving isolates with larger pycnidia greater epidemic potential, with more spores produced during each cycle of infection. In breeding programs, resistant cultivars are typically selected primarily based on the presence of leaf lesions or necrosis. In our dataset, some cultivars and genotypes showed high levels of leaf lesions but relatively low numbers of pycnidia. Because pycnidia represent a direct measure of pathogen reproductive output and pycnidia density likely reflects the epidemic potential of the pathogen on a particular host genotype, accurate measurements of pycnidia production may provide a better indication of host resistance.

Melanin is a dark pigment produced by a wide range of microbes, including plant pathogens. Melanization in fungi has been linked to several agronomically relevant traits, including stress tolerance (Singaravelan et al. 2008) and virulence (Scharf et al. 2014). By using gray values extracted from 8-bit grayscale images, we were able to obtain a proxy for melanization based on the assumption that darker-colored pycnidia contain more melanin. In mapping populations generated using controlled crosses, melanization in Z. tritici was shown to be a quantitative trait in vitro (Lendenmann et al. 2014). Pycnidia size was also shown to be a quantitative trait in the same mapping populations using infections on two wheat cultivars conducted under highly controlled greenhouse conditions (Stewart and McDonald 2014). The data presented here show that this is also the case in natural field infections encompassing many different host backgrounds and different environmental conditions. In this study, significant differences in pycnidia size and melanization were found between cultivars. Assuming that a relatively homogeneous, though genetically diverse, population of $Z$. tritici exists across the sampled plots at both locations, this finding shows that the host influences not only the number and size of pycnidia but also the level of melanin produced by the pathogen.

Despite regular fungicide applications throughout the growing season, high levels of $Z$. tritici infection were observed on treated plants in both locations. Swiss plots were treated primarily with azole (demethylation inhibitor [DMI]) fungicides (spiroxamine, prothioconazole,fenpropidin, and tebuconazole), with one application containing a mixture of prothioconazole and bixafen from the succinate dehydrogenase inhibitor (SDHI) class of fungicides. Resistance to DMI fungicides in Europe is widespread (Leroux and Walker 2011) and has previously been reported in Swiss Z. tritici populations (Brunner et al. 2008). Although less widespread than DMI resistance, reduced sensitivity and resistance to SDHI fungicides has also been reported (Sierotzki and Scalliet 2013; Torriani et al. 2015). Oregon plots were treated with azoxystrobin from the quinone outside inhibitor (QoI) fungicide class. Resistance to QoI fungicides was first observed in North America in 2012 in isolates collected from western Oregon (Estep et al. 2013) and has since been shown to be under selection (Hayes et al. 2016). Given the known resistance of $Z$. tritici populations to widely used fungicides, it is not surprising that significant levels of STB were found in treated fields in both locations. Interestingly, other common fungal pathogens were rarely found on the treated plants in either location. This observation further highlights the increasing problem of fungicide resistance in natural Z. tritici populations but also illustrates how the high level of fungicide resistance in natural populations can now be used as a tool to study STB in a nearly pure culture under field conditions, enabling an efficient measurement of differences in quantitative resistance among wheat lines and cultivars.

Different overall levels of STB disease were observed at the two sampling locations. Different environmental conditions, pathogen populations, and agronomic practices were present at each location 
and could account for the observed differences but other factors may also play a role, including genetic differences associated with the two plant populations and different sampling protocols. The Oregon population comprised a series of RILs generated from two wheat cultivars whereas the Swiss population comprised a diverse group of widely grown cultivars and likely had a wider base of genetic diversity. Despite these host differences, there was more variance in disease phenotypes in the Oregon population than the Swiss population. This may reflect differences in leaf sampling protocols. The RIL lines were chosen based on visible differences in disease severity while the Swiss cultivars were not. In addition, the flag leaf was systematically sampled in the Oregon population whereas, in Switzerland, the plants were less mature and leaves typically were sampled one or two leaves below the flag leaf. Finally, a smaller number of host genotypes were sampled in Oregon compared with Switzerland. It is clear that a consistent method of sampling will need to be developed to enable more accurate comparisons of quantitative resistance between experiments and locations.

For the Swiss cultivars, there was little agreement between the existing disease ratings and the observed phenotypes. However, in the Oregon population, there was a strong correlation between field scores and PLACL, pycnidia count, and pycnidia per square centimeter of leaf. Field scores were made by visually assessing the percentage of diseased tissue within plots; therefore, it is not surprising that this correlated well with PLACL from image analysis. From the image analysis, it is also evident that a strong correlation exists between PLACL and both pycnidia count and pycnidia per square centimeter of leaf. Therefore, it follows that pycnidia count and pycnidia per square centimeter of leaf would also correlate with the field scores.

The method of counting pycnidia using automated image analysis was shown to be accurate when compared with true values based on manual counts. Image analysis was previously shown to be more accurate than visual estimation in several pathosystems (Bock et al. 2008; Kokko et al. 2000; Martin and Rybicki 1998; Xie et al. 2012). Field plots are most commonly assessed for disease levels using visual estimates. Although this method reduces the subjectivity associated with visual estimates, some subjectivity remains when comparing image analysis with manual counts. In some cases, it is difficult to ascertain which features on a leaf are actually pycnidia. An advantage of image analysis is that any variation will be consistent through time because computers are not subject to subjectivity and fatigue like human scorers. The ability to accurately dissect the various components of $Z$. tritici virulence in the field will enable researchers and breeders to more accurately assess virulence in the pathogen and resistance in the host.

Field estimates of STB severity based on PLACL can be accomplished in approximately $30 \mathrm{~s} /$ plot (C. C. Mundt and C. H. Hagerty, unpublished), allowing a large number of host genotypes to be assessed very quickly. This can be very important in a breeding program, because the number of progeny assessed is closely related to breeding progress. Although the time needed to preprocess and scan the leaves will be longer than field-based visual estimates, the use of image analysis has several benefits. The work can be carried out by more than one person without any risk of variation between people, as is the case with visual estimates. Collection and mounting of infected leaves can be carried out by unskilled workers with minimal instruction. The ability to dissect different aspects of the disease (e.g., leaf damage versus pathogen reproduction) into leaf symptoms as well as provide a direct measure of pathogen reproduction provides a more accurate representation of the disease. It is already clear that some isolate-cultivar interactions produce high levels of leaf lesions but relatively few pycnidia (Stewart and McDonald 2014). Therefore, making an assessment of pathogen virulence or host resistance based solely on leaf lesions or necrosis may not give a complete picture. Unlike field estimates, images can be stored and referred back to in the future should anomalies be identified.
There was no difference in the phenotypes measured by two different scanners from two different manufactures except for pycnidia gray value. Therefore, comparisons between datasets made with different hardware can be considered valid, with the exception of pycnidia melanization, where caution should be exercised. From the measurements of black reference points and blank areas, it appears that the Epson scanner was able to capture a significantly wider range of values, which resulted in the overall lower pycnidia gray values. It is unclear where these differences arise but possible explanations include differences in the light source, sensors, or interpretation of values into visible images.

Feedback from users of the original image analysis macro (Stewart and McDonald 2014) highlighted areas that required improvement to enhance the usability of the system. The implementation of a QR code reader and a custom results table that includes sample names and relative measures such as pycnidia density means that the output can be used directly without any additional manipulation. The use of a flatbed scanner eliminates variation in lighting and camera settings. The ability to scan eight leaves at one time also reduces operator input and time needed to generate a dataset. Comprehensive instructions were provided to help others utilize the method. This has resulted in a method that is easier to use, more consistent in outputs, and requires less pre- and postprocessing time. The ever-decreasing costs of hardware and data storage mean that, in contrast to more sophisticated phenotyping systems, this method can be implemented with minimal capital investment. The results presented here could be obtained using equipment costing $<\$ 1,000$.

Accurate assessment of plant disease under field conditions is key to making progress in several areas of plant pathology. The methods presented allow accurate measurements of different symptom components, including plant damage, represented by the percentage of leaf area covered by lesions, as well as direct measures of pathogen reproduction, represented by the number of fruiting bodies produced on a leaf. We believe that this new ability to accurately measure different quantitative components of disease under field conditions will assist resistance breeding efforts as well as provide novel insights into pathogen biology. The method described here is currently applicable only to Z. tritici on wheat but it has the potential to be adapted to other pathosystems. We provided all files necessary to use the method (as supplementary material and also available for download as a single file from http://www.path. ethz.ch/links/pathology.html) and encourage others to adapt the method to meet their needs and to share their modifications with the plant pathology community. Our results show that STB resistance to natural infections under field conditions is quantitative and comprises several components. It remains to be determined how best to combine these resistance components to optimize progress in breeding wheat cultivars that are more resistant to STB.

\section{ACKNOWLEDGMENTS}

This research was supported by a grant from the Swiss National Science Foundation (31003A_134755). B. A. McDonald acknowledges receipt of a fellowship in 2015 from the OECD Co-operative Research Program: Biological Resource Management for Sustainable Agricultural Systems while on sabbatical at Oregon State University. A. Mikaberidze was supported by the ERC advanced grant PBDR 268540. K. E. Sackett and M. Dolores Vazquez assisted with leaf collection and E. M. Sykes assisted with leaf preparation in Oregon. We thank the crop science group of the ETH Zürich (http://www.kp.ethz.ch) for granting access to the wheat association panel at the Field Phenotyping Platform. L. Meile and E. Morf assisted with leaf preparation in Switzerland.

\section{LITERATURE CITED}

Abd-El-Haliem, A. 2012. An unbiased method for the quantitation of disease phenotypes using a custom-built macro plugin for the program ImageJ. Pages 635-644 in: Plant Fungal Pathogens. Methods in Molecular Biology, Vol. 835. M. D. Bolton and B. P. H. J. Thomma, eds. Humana Press, New York. 
Bock, C. H., Parker, P. E., Cook, A. Z., and Gottwald, T. R. 2008. Visual rating and the use of image analysis for assessing different symptoms of citrus canker on grapefruit leaves. Plant Dis. 92:530-541.

Bock, C. H., Poole, G. H., Parker, P. E., and Gottwald, T. R. 2010. Plant disease severity estimated visually, by digital photography and image analysis, and by hyperspectral imaging. Crit. Rev. Plant Sci. 29:59-107.

Brown, J. K. M., Chartrain, L., Lasserre-Zuber, P., and Saintenac, C. 2015. Genetics of resistance to Zymoseptoria tritici and applications to wheat breeding. Fungal Genet. Biol. 79:33-41.

Brunner, P. C., Stefanato, F. L., and McDonald, B. A. 2008. Evolution of the CYP51 gene in Mycosphaerella graminicola: Evidence for intragenic recombination and selective replacement. Mol. Plant Pathol. 9:305-316.

Courvoisier, N., Häner, L. L., Bertossa, M., Thévoz, E., Anders, M., Stoll, P., Weisflog, P., Dugon, J., and Grünig, K. 2015. Liste recommandée des variétés de céréales pour la récolte 2016. Online publication. Recherche Agronomique Suisse. http://www.swissgranum.ch/88-2-Listes-recommandes.html

Cowger, C., and Mundt, C. C. 2002. Aggressiveness of Mycosphaerella graminicola isolates from susceptible and partially resistant wheat cultivars. Phytopathology 92:624-630.

Dean, R., Van Kan, J. A. L., Pretorius, Z. A., Hammond-Kosack, K. E., Di Pietro, A., Spanu, P. D., Rudd, J. J., Dickman, M., Kahmann, R., Ellis, J., and Foster, G. D. 2012. The top 10 fungal pathogens in molecular plant pathology. Mol. Plant Pathol. 13:413-430.

de Mendiburu, F. 2014. Agricolae: Statistical procedures for agricultural research. R package version 1.1-8. Online publication. https://cran.r-project. org/web/packages/agricolae/index.html

Estep, L. K., Zala, M., Anderson, N. P., Sackett, K. E., Flowers, M., McDonald, B. A., and Mundt, C. C. 2013. First report of resistance to QoI fungicides in North American populations of Zymoseptoria tritici, causal agent of Septoria tritici blotch of wheat. Plant Dis. 97:1511.

Fones, H., and Gurr, S. 2015. The impact of Septoria tritici blotch disease on wheat: An EU perspective. Fungal Genet. Biol. 79:3-7.

Gabrielson, J., Hart, M., Jarelöv, A., Kühn, I., McKenzie, D., and Möllby, R. 2002. Evaluation of redox indicators and the use of digital scanners and spectrophotometer for quantification of microbial growth in microplates. J. Microbiol. Methods 50:63-73.

Green, J. M., Appel, H., Rehrig, E. M., Harnsomburana, J., Chang, J.-F., Balint-Kurti, P., and Shyu, C.-R. 2012. PhenoPhyte: A flexible affordable method to quantify 2D phenotypes from imagery. Plant Methods 8:45.

Hagerty, C. H., and Mundt, C. C. 2015. Zymoseptoria tritici fungicide resistance dynamics: Seasonal changes and effects on virulence. Page 41 in: Resistance 2015. Rothamsted Research, Harpenden, Hertfordshire, UK.

Hayes, L. E., Sackett, K. A., Anderson, N. P., Flowers, M. D. and Mundt, C. C. 2016. Evidence of selection for fungicide resistance in Zymoseptoria tritici populations on wheat in western Oregon. Plant Dis. 100:483-489.

Jørgensen, L. N., Hovmøller, M. S., Hansen, J. G., Lassen, P., Clark, B., Bayles, R., Rodemann, B., Flath, K., Jahn, M., Goral, T., Jerzy Czembor, J., Cheyron, P., Maumene, C., De Pope, C., Ban, R., Nielsen, G. C., and Berg, G. 2014. IPM strategies and their dilemmas including an introduction to www.eurowheat.org. J. Integr. Agric. 13:265-281.

Kato, Y., Okami, M., Tajima, R., Fujita, D., and Kobayashi, N. 2010. Root response to aerobic conditions in rice, estimated by Comair root length scanner and scanner-based image analysis. Field Crops Res. 118:194-198.

Kokko, E. G., Conner, R. L., Lee, B., Kuzyk, A. D., and Kozu, G. C. 2000. Quantification of common root rot symptoms in resistant and susceptible barley by image analysis. Can. J. Plant Pathol. 22:38-43.

Kwack, S. M., Kim, N. E., Lee, H., Kim, J.-W., Chun, S.-C., and Kim, D. K. 2005. Digital image analysis to measure lesion area of cucumber anthracnose by Colletotrichum orbiculare. J. Gen. Plant Pathol. 71:418-421.

Lamari, L. 2002. ASSESS: Image Analysis Software for Plant Disease Quantification. American Phytopathological Society Press, St. Paul, MN.

LaTeX3 Project Team. 2015. LaTeX-A document preparation system. Online publication. https://latex-project.org

Lendenmann, M. H., Croll, D., Stewart, E. L., and McDonald, B. A. 2014. Quantitative trait locus mapping of melanization in the plant pathogenic fungus Zymoseptoria tritici. G3: Genes Genomes Genet. 412:2519-2533.
Leroux, P., and Walker, A.-S. 2011. Multiple mechanisms account for resistance to sterol $14 \alpha$-demethylation inhibitors in field isolates of Mycosphaerella graminicola. Pest Manage. Sci. 67:44-59.

Lin, L. I. K. 1989. A concordance correlation coefficient to evaluate reproducibility. Biometrics 45:255-268.

Mahlein, A. 2016. Plant disease detection by imaging sensors-Parallels and specific demands for precision agriculture and plant phenotyping. Plant Dis. 100:241-251.

Maloof, J. N., Nozue, K., Mumbach, M. R., and Palmer, C. M. 2013. LeafJ: An ImageJ plugin for semi-automated leaf shape measurement. J. Vis. Exp. 71: e50028.

Martin, D. P., and Rybicki, E. P. 1998. Microcomputer-based quantification of Maize streak virus symptoms in Zea mays. Phytopathology 88:422-427.

Mundt, C. C. 2014. Durable resistance: A key to sustainable management of pathogens and pests. Infect. Genet. Evol. 27:446-455.

Mutka, A. M., and Bart, R. S. 2014. Image-based phenotyping of plant disease symptoms. Front. Plant Sci. 5:734.

O’Driscoll, A., Kildea, S., Doohan, F., Spink, J., and Mullins, E. 2014. The wheat-Septoria conflict: A new front opening up? Trends Plant Sci. 19: 602-610.

Orton, E. S., Deller, S., and Brown, J. K. 2011. Mycosphaerella graminicola: From genomics to disease control. Mol. Plant Pathol. 12:413-424.

Pethybridge, S. J., and Nelson, S. C. 2015. Leaf Doctor: A new portable application for quantifying plant disease severity. Plant Dis. 99:13101316.

R Core Team. 2012. R: A Language and Environment for Statistical Computing. Online publication. R Foundation for Statistical Computing, Vienna. http://www.R-project.org/

Rasband, W. S. 1997-2015. ImageJ. Online publication. U.S. National Institutes of Health, Bethesda, MD. http://imagej.nih.gov/ij/

Scharf, D. H., Heinekamp, T., and Brakhage, A. A. 2014. Human and plant fungal pathogens: The role of secondary metabolites. PLoS Pathog. 10: e1003859.

Sierotzki, H., and Scalliet, G. 2013. A review of current knowledge of resistance aspects for the next-generation succinate dehydrogenase inhibitor fungicides. Phytopathology 103:880-887.

Singaravelan, N., Grishkan, I., Beharav, A., Wakamatsu, K., Ito, S., and Nevo, E. 2008. Adaptive melanin response of the soil fungus Aspergillus niger to UV radiation stress at Evolution Canyon, Mount Carmel, Israel. PLoS One 3:e2993.

Stevenson, M., Nunes, T., Heuer, C., Marshall, J., Sanchez, J., Thornton, R., Reiczigel, J., Robison-Cox, J., Sebastiani, P., Solymos, P., and Yoshida, K. 2013. epiR: An R package for the analysis of epidemiological data. R package version 0.9-48. https://cran.r-project.org/web/packages/epiR/index.html

Stewart, E. L., and McDonald, B. A. 2014. Measuring quantitative virulence in the wheat pathogen Zymoseptoria tritici using high-throughput automated image analysis. Phytopathology 104:985-992.

Suffert, F., Sache, I., and Lannou, C. 2013. Assessment of quantitative traits of aggressiveness in Mycosphaerella graminicola on adult wheat plants. Plant Pathol. 62:1330-1341.

Torriani, S. F. F., Melichar, J. P. E., Mills, C., Pain, N., Sierotzki, H., and Courbot, M. 2015. Zymoseptoria tritici: A major threat to wheat production, integrated approaches to control. Fungal Genet. Biol. 79:8-12.

Vazquez, M. D., Zemetra, R., Peterson, C. J., Chen, X., Heesacker, A., and Mundt, C. 2015. Multi-location wheat stripe rust QTL analysis: Genetic background and epistatic interactions. Theor. Appl. Genet. 128:1307-1318.

Xie, W., Yu, K., Pauls, K. P., and Navabi, A. 2012. Application of image analysis in studies of quantitative disease resistance, exemplified using common bacterial blight-common bean pathosystem. Phytopathology 102: 434-442.

Zhan, J., Mundt, C. C., Hoffer, M. E., and McDonald, B. A. 2002. Local adaptation and effect of host genotype on the rate of pathogen evolution: An experimental test in a plant pathosystem. Evol. Biol. 15:634-647.

Zhan, J., Torriani, S. F. F., and McDonald, B. A. 2007. Significant difference in pathogenicity between MAT1-1 and MAT1-2 isolates in the wheat pathogen Mycosphaerella graminicola. Fungal Genet. Biol. 44:339-346. 